\title{
Interaction Opportunities Around Helmet Design
}

Wouter Walmink

Exertion Games Lab

RMIT University

Melbourne, Australia

wouter@exertiongameslab.org

\section{Alan Chatham}

Exertion Games Lab

RMIT University

Melbourne, Australia

wouter@exertiongameslab.org

Florian 'Floyd' Mueller

Exertion Games Lab

RMIT University

Melbourne, Australia

floyd@exertiongameslab.org

Permission to make digital or hard copies of part or all of this work for personal or classroom use is granted without fee provided that copies are not made or distributed for profit or commercial advantage and that copies bear this notice and the full citation on the first page. Copyrights for third-party components of this work must be honored. For all other uses, contact the Owner/Author.

Copyright is held by the owner/author(s)

CHI 2014, Apr 26 - May 01 2014, Toronto, ON, Canada

ACM $978-1-4503-2474-8 / 14 / 04$

ACM 978-1-4503-2474-8/14/04.

\begin{abstract}
People wear helmets to protect themselves in case of accidents. We are asking: what other purposes could a helmet serve? Our answer LumaHelm turns the helmet into a display for communication, expression and play. LumaHelm allows us to explore how to support cyclists safety, skateboarders' self-expression and riders' communication of heart rate. Our work demonstrates the feasibility of helmets as platform for mounting interactive technology. It also highlights a new interaction technique, possible when the data on wearable displays is available to others nearby, but not to the wearer. With our work, we aim to inspire fellow design researchers to consider how safety gear like helmets can use interactivity technology to support the activities in which they are used.
\end{abstract}

\section{Author Keywords}

Wearables; heart rate; biofeedback; body-worn display; exertion interface; cycling; helmet

\section{ACM Classification Keywords}

H.5.2 Information Interfaces and Presentation: User Interfaces 


\section{Introduction}

Helmets can help people reduce physical injury during potentially dangerous activities like cycling, skating and rock-climbing. Accidents are nevertheless relatively uncommon, so many consider helmets more of an inconvenience or burden. This begs the question what can helmets do for us besides protecting us from physical harm? Our response to this question is LumaHelm (Figure 1), an inspirational interactive design prototype that explores helmets as interaction platform. LuamHelm may not only make the associated physical activity safer, but also help to make wearing the helmet more engaging, and possibly even fun.

\section{LumaHelm}

Previous research has explored various ways in which wearable interactive technology can augment physical activity, for example see the many mobile phone sports apps. We noticed that there is little work on helmetbased concepts, and if so, they focus on the interaction between the helmet and the wearer $[5,8]$. In response we developed a helmet where the interaction is also oriented outward, and covered an existing bicycle helmet with a total of 104 individually addressable RGB LEDs. The LEDs are controlled by an Arduino Uno board. Because the memory and processing power of the Arduino is limited, and to simplify the control of our system, we developed a library in Processing allowing us to instantly map what we draw on a computer screen to the lights on the helmet. This setup allows anyone with minimal programming experience to create rich visuals with our helmet, and control them through any sensors that can interface with the Arduino or any computer.

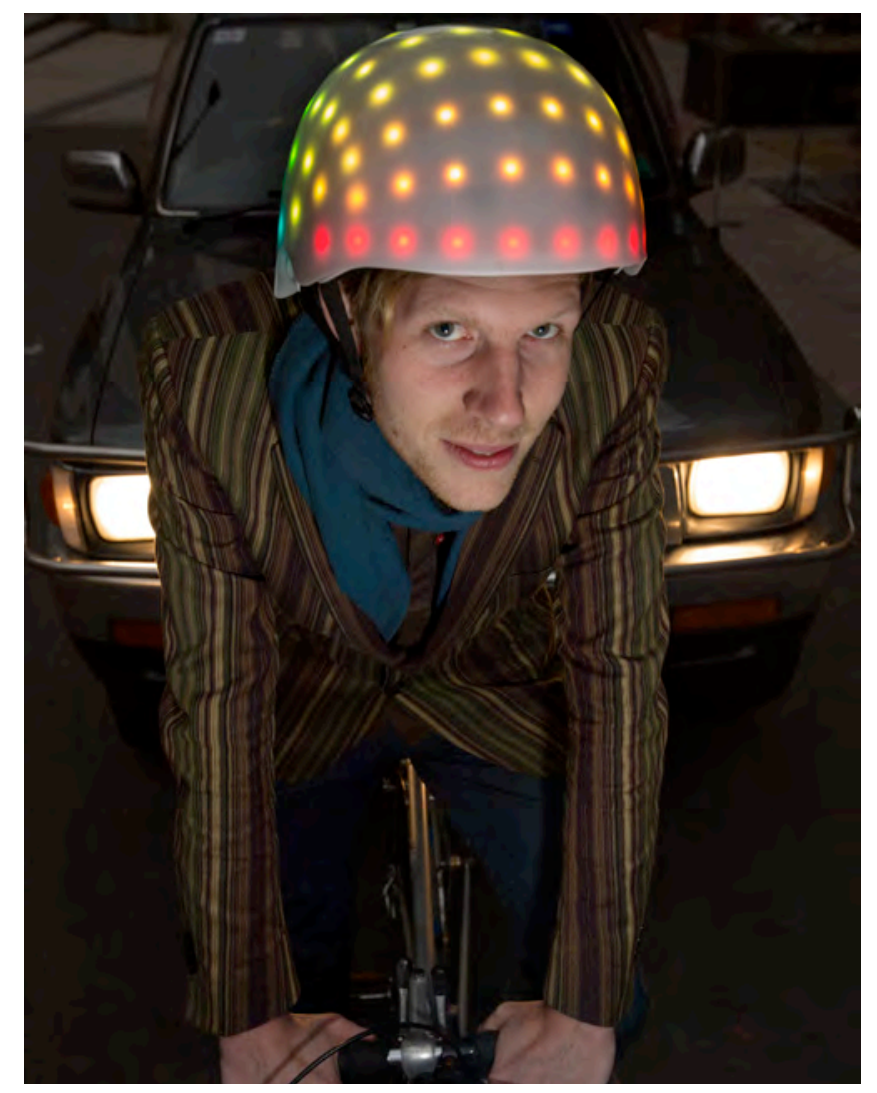

Figure 1. LumaHelm.

\section{Construction}

We wanted to maintain the structural integrity of the helmet as much as possible, so we glued the LED strips to the outside of an existing helmet, and designed a new cover to be fitted over the electronics. To create this new exterior shell, we made a 3D scan of the helmet and modeled a slightly larger version (1) that 
was then CNC-milled in layers from a block of wood

(2). The layers were sanded down into a smooth mold (3) out of which we made a vacuum-formed cast that we layered over the LED strips (4), see Figure 2.

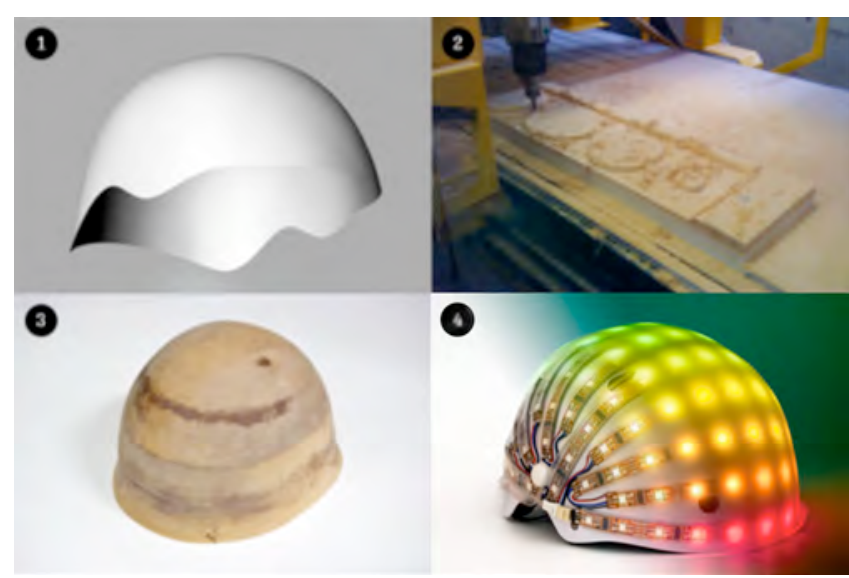

Figure 2. Lower right is a composite image, showing LumaHelm's interior (left) and exterior (right) design

\section{Interactive concept demonstrations}

In an effort to exemplify some of the ways in which LumaHelm could be used in various forms of sports and physical activity, we have built the following demonstrations.

LumaHelm as utilitarian device

An accelerometer embedded in the helmet allows cyclists trigger the LEDs to light up up as an indicator by tilting their head in the respective direction. The back of the helmet can also turn bright red in response to a head movement (or alternatively, triggered through usage of the brakes), warning people behind of a braking maneuver.
LumaHelm supporting bodily self-expression

The same accelerometer can support skateboarders in their bodily self-expression when doing skateboarding tricks. LumaHelm lights up in various animated colors based on the speed, jumps and turns a skateboarder makes. This can also provide additional entertainment and spectacle for audiences watching the performance.

\section{LumaHelm as an artistic medium}

LumaHelm can sense audio data through a microphone using the data to drive visualizations. We implemented a visual equalizer, responding to the dramatic play of a guitarist. In the dimmed context of a concert hall, the resulting light may not only be seen by the audience, but even indirectly by the wearer, as light bounces of near surroundings.

LumaHelm communicating bodily data

Connecting the system to a heart rate sensor, we visualized the wearer's heart rate as a pulsating light. By making other road users' more aware of a cyclist's physiological state, we see an opportunity to contribute to an increased understanding (and appreciation) of each other on the road, an approach also proposed by Ducao et al. [2].

\section{Contributions}

With LumaHelm, we are making two key contributions:

Protective gear as platform for interactive technology We show protective gear is a viable mounting platform for interactive technology. Helmets and other protective wearable gear [3] offer unique interaction opportunities as they are "wearables" that are worn close to the body [6]. However, traditional wearables are often built into clothing, creating challenges for the designer. For 
example, users may want to wear multiple layers of clothing, causing occlusion issues. Furthermore, attaching displays to fabric can be difficult [7]. While dedicated technology solutions aim to address this [1] we point out that protective gear such as helmets bypass many of these issues, as they have structures with hard surfaces that lend themselves to technology mounting $[5,8]$. The implemented examples discussed in this paper have received positive attention from media as well as groups and individuals involved in activities that require helmets. This suggests to us that the combination of interactive technology and helmets is an area deserving further investigation.

Wearable displays afford unique information access LumaHelm shows that attaching a display to the body makes it easy for people in close physical proximity to see displayed information, yet hard for the wearer. This interaction technique stands in contrast to the experience with most mobile devices that display personal information. For example, social media integration in many mobile apps makes access to personal information easy for people far away, yet the private nature and size of mobile phone screens limits access to people close-by. LumaHelm points out that these three levels of access to personal information-by the wearer, people in close physical proximity, and people out of sight-can be influenced by the mounted location of the display, adding to our understanding of the notion of proxemics [4].

\section{Conclusion}

We presented LumaHelm, an interactive helmet featuring an LED display fuelled by sensor data to explore interaction techniques around wearable safety gear. With our work, we aim to inspire fellow design researchers to consider how safety gear like helmets can be augmented to support the activities in which they are used.

\section{Acknowledgements}

We thank the members of the Exertion Games Lab, the communications office at RMIT University and Bicycle Victoria. Florian 'Floyd' Mueller is the recipient of an Australian Research Council Fellowship (DP110101304).

\section{References}

1. Lilypad Arduino. http://lilypadarduino.org/.

2. MindRider.

http://web.media.mit.edu/ arlduc/projects/mindrid er/.

3. Chi, E.H., Song, J. and Corbin, G. "Killer App" of wearable computing: wireless force sensing body protectors for martial arts. Proc. UIST'04, ACM, 277285.

4. Greenberg, S., Marquardt, N., Ballendat, T., DiazMarino, R. and Wang, M. Proxemic interactions: the new ubicomp? interactions, 18 (1). (2011), 42-50.

5. Jones, E.M., Selker, T. and Chung, H., What you said about where you shook your head: a handsfree implementation of a location-based notification system. CHI'O7 Extended Abstracts, ACM, 24772482.

6. Mann, S. Wearable computing: A first step toward personal imaging. Computer, 30 (2). (1997), 25-32.

7. Page, M. and Moere, A.V. Evaluating a wearable display jersey for augmenting team sports awareness Pervasive Computing, Springer, 2007, 91-108.

8. Stern, B. Citi Bike Helmet, 2013. http://learn.adafruit.com/citi-bike-helmet/. 